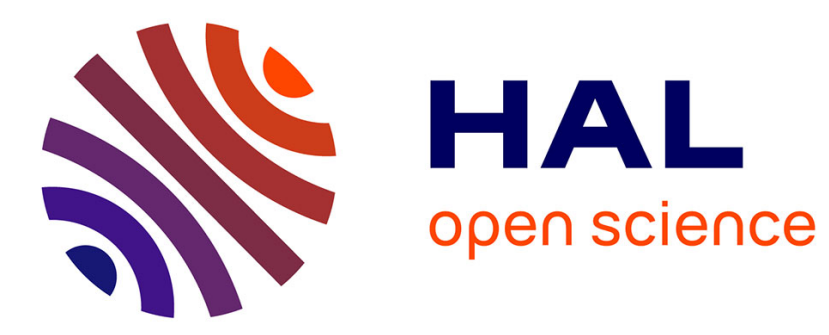

\title{
ADN, métabolisme et individualité dans la biologie philosophique de Hans Jonas
}

\author{
Sonia Dheur, Sven J Saupe
}

\section{To cite this version:}

Sonia Dheur, Sven J Saupe. ADN, métabolisme et individualité dans la biologie philosophique de Hans Jonas. Bulletin d'histoire et d'épistémologie des sciences de la vie , 2019, 26 (2), pp.223-238. 10.3917/bhesv.262.0223 . halshs-02283879

\section{HAL Id: halshs-02283879 \\ https://shs.hal.science/halshs-02283879}

Submitted on 16 Dec 2020

HAL is a multi-disciplinary open access archive for the deposit and dissemination of scientific research documents, whether they are published or not. The documents may come from teaching and research institutions in France or abroad, or from public or private research centers.
L'archive ouverte pluridisciplinaire HAL, est destinée au dépôt et à la diffusion de documents scientifiques de niveau recherche, publiés ou non, émanant des établissements d'enseignement et de recherche français ou étrangers, des laboratoires publics ou privés. 
ADN, métabolisme et individualité dans la biologie philosophique de Hans Jonas

Sonia Dheur ${ }^{1}$ et Sven J. Saupe ${ }^{2}$

${ }^{1}$ Passages

UMR5319 CNRS - Université Bordeaux Montaigne

Maison des Suds

12 esplanade des Antilles

33607 Pessac, France

téléphone : +33556848212

courrier électronique : sonia.dheur@cnrs.fr

(pour correspondance)

${ }^{2}$ Reconnaissance du non-soi chez les champignons

Institut de Biochimie et Génétique Cellulaires

UMR5095 CNRS - Université de Bordeaux

1 rue Camille Saint Saëns

33077 Bordeaux, France

téléphone : +33556999027

courrier électronique : sven.saupe@ibgc.cnrs.fr 


\section{Summary}

In The Phenomenon of Life, Hans Jonas introduces an ontology of organic life based on the notion of needful freedom centered on the exercise of metabolic activity. In a brief appendix to the third essay of this collection Jonas poses that DNA and neural cells of higher animals are excluded from metabolism and therefore constitute exceptional material invariants. We attempt here to analyse this appendix and related texts to delimit the outlines of the Jonassian understanding of organic life. We propose that in contrast to the claim developed in the appendix (but in full agreement with Jonas' general line of thought) neither DNA nor neural cells can be considered as strict material invariants. We then go on to discuss the deflation in these pages, of certain aspects of organic phenomena such as growth, development and foremost evolution, in favor of what has been previously described as an "embodied teleology". We contend that, with Jonas, an articulation of the biological and phenomenological view of the living processes is both possible and fruitful.

\section{Résumé}

Dans le phénomène de la vie, Hans Jonas introduit une ontologie de la vie organique comme l'exercice d'une liberté nécessiteuse centrée sur le métabolisme. Dans un bref appendice du troisième essai qui compose l'ouvrage, Jonas pose que l'ADN et les cellules neuronales seraient exclus du métabolisme et constitueraient ainsi des exceptions comme invariants matériels. Nous analysons ce texte pour tenter de préciser la conception jonassienne de la vie organique. Nous posons ainsi, qu'à contre-courant de cet appendice (mais en accord avec la pensée générale de Jonas), ni l'ADN, ni les neurones ne peuvent être considérés comme des invariants matériels stricts. Nous soulignons aussi la déflation, dans cet appendice, de certains aspects de la vie organique comme la croissance, le développement et surtout l'évolution au profit d'une prise en compte de l'organisme immédiat. Nous proposons qu'avec Jonas une articulation des perspectives biologiques et phénoménologiques sur les processus du vivant soit à la fois possible et féconde.

\section{Auteurs}

Sonia Dheur est biologiste moléculaire, chargée de recherche CNRS à l'UMR 5319 Passages (InSHS), et s'intéresse notamment à la construction des savoirs et à la circulation des imaginaires en société.

Sven J. Saupe, généticien, directeur de recherche CNRS à 1'UMR 5095 IBGC (InSB), travaille notamment sur la reconnaissance du soi et du non-soi chez les champignons. 
Le phénomène de la vie apparaît comme le maître-ouvrage de la biologie philosophique de Hans Jonas et servira comme ontologie du vivant à la fondation de son éthique développée dans Le Principe Responsabilité. L'ouvrage correspond à une collection d'essais qui s'inscrit dans un projet de philosophie de la vie visant à inclure dans une même perspective «philosophie de l'organisme et philosophie de l'esprit »' selon une tradition qui remonte à la Naturphilosophie et à l'idéalisme allemand $^{2}$. L'essai clef de cette collection porte le titre de «Dieu est-il mathématicien? La signification du métabolisme ». Il fait suite à deux essais portant l'un sur la trajectoire historique de l'appréhension du vivant (allant d'un monisme animiste au dualisme cartésien puis au-delà) ; l'autre sur les conséquences philosophiques radicales du darwinisme. C'est le troisième essai, souvent repris, qui pose une ontologie du vivant. Il est construit comme un questionnement de l'expérience phénoménale du vivant que pourrait avoir un Dieu omniscient et mathématicien. L'argument est que ce Dieu manquerait le vivant faute de n'être vivant lui-même. Une idée que Merleau-Ponty formule par «je sais l'organisme parce que je le suis» ${ }^{3}$ (qui rejoint le «La pensée du vivant doit tenir du vivant l'idée du vivant » de Canguilhem ${ }^{4}$ ). D'une certaine façon, l'essai est lié à la critique kantienne de la compréhension du vivant qui ne pourrait être abordée comme une démarche logico-mathématique et qui pose que jamais un «Newton du brin d'herbe » ne pourra émerger ${ }^{5}$. L'essai se concentre, c'est son originalité, sur le processus du métabolisme et ce qui est décrit comme l'ontologie spécifique du vivant, « un mode d'être remarquable ${ }^{6}$, par lequel l'identité matérielle de l'organisme ne coïncide pas avec l'identité du tout de l'organisme. C'est seulement le flux constant de matière à travers l'organisme qui permet le maintien de sa forme. Le moment où forme et matière viennent à coïncider signe la mort de l'organisme. Le métabolisme, cet échange de matière entre l'organisme et le monde est la modalité par laquelle s'établit la liberté nécessiteuse (needful freedom) du vivant :

Dénotant, du côté de la liberté, une capacité de la forme organique, celle de changer sa matière, le métabolisme dénote également la nécessité irrémédiable pour elle de le faire. Son "pouvoir » est un «devoir », puisque son exécution est identique à son être. Elle peut, mais elle ne peut cesser de faire ce qu'elle peut sans cesser d'être ${ }^{7}$.

\footnotetext{
${ }^{1}$ H. Jonas, Le phénomène de la vie. Vers une biologie philosophique, Bruxelles, de boeck, 2001, p.13.

${ }^{2}$ F. Beiser, "Hegel and Naturphilosophie", Studies in History and Philosophy of Science, 2003, 34, 135-147.

${ }^{3}$ M. Merleau-Ponty, Notes de cours 1959-1961, Paris, Gallimard, 1996, p.387, cité par J. Farges, 2010.

${ }^{4}$ G. Canguilhem, La connaissance de la vie, Paris, Vrin, 2015, p.16.

${ }^{5}$ E. Kant, Critique de la faculté de juger, Paris, Gallimard, 1985, p.369. ; P. Schuster, "Is there a Newton of the blade of grass ?", Complexity, 2011, 16(6), 5-9; A. Weber and F.J. Varela, "Life after Kant: Natural purpose and autopoietic foundations of biological individuality". Phenomenology and the Cognitive Sciences 2002, 1, 97-125.

${ }^{6}$ H. Jonas, p. 86, op. cit.

${ }^{7}$ H. Jonas, p. 93, op. cit.
} 
Cette même ontologie qui place l'activité métabolique au centre de la compréhension du vivant est aussi développée dans un texte légèrement plus tardif ${ }^{8}$. L'appréhension du vivant comme « souci », comme liberté nécessiteuse, avec cette non-coïncidence métabolique de la matière et de la forme, et l'articulation d'un soi au monde y sont développées de nouveau, mais sous une forme différente qui s'écarte de l'expérience de pensée et construit l'argumentation autour de la notion d'individu. On peut noter dès à présent avec Simondon et Jonas lui-même que ce schème hylémorphique de l'individu («considérant l'individu comme engendré par la rencontre d'une forme et d'une matière ») ${ }^{9}$ s'applique aussi aux objets techniques et non aux seuls vivants. Ainsi, il est requis de poser d'une part la nécessité du flux de matière à travers la forme pour le maintien de l'être et d'autre part le caractère immanent de ce remplacement pour rendre compte de l'individualité dans le vivant.

L'essai III du Phénomène de la vie est complété par trois brefs appendices, le premier portant sur le rôle des mathématiques dans les sciences naturelles dans l'Antiquité grecque, le deuxième sur la philosophie de l'organisme chez Whitehead et le troisième qui nous intéresse ici, sur « la nonparticipation de l'ADN au métabolisme $»^{10}$. Ce curieux petit appendice figure dans l'édition anglaise mais plus dans deux traductions ultérieures allemandes, et ce pour des raisons inconnues ${ }^{11}$. Ce texte relève que la proposition développée dans l'essai selon laquelle «aucun substrat inerte [...] n'est disponible comme référent pour une identité externe » doit être nuancé puisque l'ADN « qui contrôle le métabolisme du reste de la cellule est lui-même exempt de métabolisme, c'est-à-dire qu'il n'est pas sujet à l'échange $»^{12}$. Jonas pose ainsi à contre-courant de sa pensée générale, l'existence d'un invariant matériel, d'un référent interne, dans le vivant (une entité qui échappe au métabolisme et où forme et matière coïncident) et se demande de quelle manière la prise en compte de cet invariant affecte l'ontologie du vivant qu'il vient de développer. Nous voudrions ici tenter d'interroger le texte de ce court appendice pour examiner les enjeux de la convocation de cette molécule dans l'ontologie du vivant de Jonas.

Une certaine phénoménologie de la vie pose de manière convaincante que la biologie ne peut rien dire de la vie concrète en raison de la radicale intériorité de cette dernière ${ }^{13}$. Cependant, il a pu être relevé qu'Husserl (dans ses travaux tardifs) ménage une place privilégiée à cette discipline scientifique comme plus proche des sources de l'évidence du monde de la vie (Lebenswelt), dans une

\footnotetext{
${ }^{8}$ H. Jonas, Les fondements biologiques de l'individualité, in Lories, Danielle, et Olivier Depré, Vie et liberté, Paris, Vrin, 2003, p.179-207.

${ }^{9}$ G. Simondon, L'individuation à la lumière des notions de forme et d'information, Grenoble, Million, 2005, p.23.

${ }^{10}$ H. Jonas, p. 107, op. cit.

${ }^{11}$ J. Urzúa, La fundamentación ontológica de la ética de la responsabilidad de Hans Jonas, thèse, Univerisdad Complutense de Madrid, 2015.

${ }^{12}$ H. Jonas, p. 107, op. cit.

${ }^{13}$ M. Henry, De la phénoménologie, Tome I, Phénoménologie de la vie Paris, Presses Universitaires de France, 2003, p.40 et p.143.
} 
attitude qui a été décrite comme un positivisme transcendantal ${ }^{14}$. Et dans cet appendice, Jonas convoque en effet des résultats de la biologie positive issus de l'étude d'une extériorité du vivant pour alimenter sa démarche phénoménologique. Ainsi, se croit-on autorisé à interroger aussi ce texte sous son aspect biologique. C'est parce que la philosophie biologique de Jonas pose comme une de ses ambitions de décrire une continuité, depuis les manifestations les plus élémentaires de la vie jusqu'à la conscience humaine, en une tentative de dépassement des impasses du dualisme cartésien, qu'il semble justifié de confronter le texte de l'auteur aux données biologiques issues de l'étude des différentes formes du vivant. Ce texte appelle de ce point de vue plusieurs questions : l'ADN est-il un invariant matériel, échappe-t-il au métabolisme et de là au principe général posé par Jonas? L'objection que Jonas se pose à lui-même est-elle justifiée ? Puis, à travers les indices présents dans ce court appendice, nous nous interrogerons sur les dimensions laissées en creux dans la phénoménologie de la vie de Jonas, notamment la dimension génétique et évolutive (mais aussi dans une moindre mesure celles du développement et de la croissance) évacuées au profit de la seule homéostasie. Ce texte court permet de saisir la situation attribuée à l'hérédité (objectivée sous la forme de l'ADN) dans son ontologie du vivant. C'est l'inflation de cette dimension (génétique et évolutive) dans la biologie moderne et sa quasi-absence dans ces pages de phénoménologie de la vie, qui par différence pourraient nous permettre de deviner comment la silhouette d'une certaine connaissance positive pourrait prendre pied sur le sol de la Lebenswelt ou comment les perspectives biologiques et phénoménologiques peuvent s'articuler.

\section{Vivant fluant ou invariant}

Dans la première partie de l'appendice, Jonas s'attache d'abord à définir le statut de l'ADN dans le cas le plus simple d'organismes unicellulaires. Il pose, contre lui-même, l'observation suivante: «Des découvertes récentes en microbiologie indiquent que l'ensemble des molécules ADN chromosomiques qui dans chaque cellule représente le code génétique, alors qu'il contrôle le métabolisme du reste de la cellule, est lui-même exempt de métabolisme, c'est-à-dire qu'il n'est pas sujet à l'échange $»^{15,16}$. Notons que Jonas fait un usage erroné - commun - de la notion de code génétique (en confondant le mode de codage en triplets avec l'information génétique proprement dite). Restons cependant, sur la simple proposition que Jonas s'oppose à lui-même, à savoir que l'ADN est exempt de métabolisme. Il existe pourtant bien un champ de la biologie qui a trait explicitement au

\footnotetext{
${ }^{14}$ J. Farges, "Vie, science de la vie et monde de la vie : sur le statut de la biologie chez le dernier Husserl", Bulletin d'analyse phénoménologique, 2010, 4 (2), 42-72.

${ }^{15}$ H. Jonas, p. 107, op. cit.

${ }^{16} \mathrm{Au}$ moment de la publication de ce texte en 1966, l'essentiel des fondements de la biologie moléculaire était élucidé. L'ADN, sa structure, sa fonction, son mécanisme de réplication et le code génétique étaient connus. De la même façon, l'usage des radioisotopes était largement développé en biologie et constituait un outil essentiel dans la mise en évidence de la nature et de l'ampleur des échanges chimiques dans le vivant (le métabolisme). En 1954, paraît dans le magazine Times un article de vulgarisation intitulé « The fleeting flesh » (la chair fuyante) qui apprend au grand public que $98 \%$ des atomes du corps humain seraient remplacés chaque année.
} 
métabolisme de l'ADN. Ce champ recouvre l'étude des mécanismes de réplication, de réparation et de modification de l'ADN. Lors de la réplication, à chaque division cellulaire, l'ADN est un bateau de Thésée dont la matière ne coïncide que pour moitié avec la forme, selon l'expression classique de réplication semi-conservative issue des expériences de Meselson et Stahl ${ }^{17}$. Jonas fait d'ailleurs allusion, comme expérience de pensée, au marquage des ADNs («si on pouvait les avoir "étiquetées" à ce moment-là en vue d'une identification ultérieure $»)^{18}$, ce qui correspond de fait à la méthode déployée dans l'expérience classique de Meselson et Stahl impliquant un marquage métabolique de l'ADN par l'azote lourd $\left({ }^{15} \mathrm{~N}\right)$. Jonas, cependant dans une note, de façon remarquable, exclut la réplication du champ de l'analyse. Il choisit de mettre à part « cet évènement reproductif » (la division cellulaire) et d'encadrer le moment du vivant entre ces deux évènements réplicatifs («qui marque respectivement son début et sa fin »), la division cellulaire qui donne naissance à l'individu et la division cellulaire qui produira deux nouveaux individus ${ }^{19}$. C'est d'abord de cette façon qu'il parvient à poser l'ADN comme exclu du métabolisme. D'autre part, il congédie également le cas de la réparation de l'ADN (« laissons de côté l'incident anormal de la mutation»), nous reviendrons sur ces deux points. Dans ce geste qui met entre parenthèses deux aspects fondamentaux de l'ADN (sa mutabilité et sa réplicabilité), Jonas pose l'ADN comme invariant non seulement dans sa matière mais aussi dans sa forme. Un troisième aspect du métabolisme de l'ADN, la modification chimique par méthylation notamment, n'est pas mentionné par Jonas. Il ne faut s'en étonner ; en effet même si ces mécanismes avaient commencé à être découverts au moment de la rédaction de ce texte, leur diffusion hors de cercles hautement spécialisés était sans doute mineure. Dans un grand nombre d'organismes, l'ADN subit lors de la vie cellulaire des modifications chimiques correspondant notamment à la méthylation de l'adénine chez les bactéries et des cytosines chez les eucaryotes. Ces modifications sont dépendantes et couplées au métabolisme du reste de la cellule et ont à leur tour un impact sur l'expression génétique qui contrôle le métabolisme cellulaire ${ }^{20}$. Ainsi lorsque Jonas écrit « le système est "clos" et son interaction avec le reste de l'organisme est simplement "catalytique" ne s'affectant pas lui-même $»^{21-22}$, il est contredit par la biologie positive actuelle. Outre ces modifications épigénétiques de l'ADN par méthylation (dont la plus grande prise en compte signe à l'heure actuelle

\footnotetext{
${ }^{17}$ M. Meselson and F.W. Stahl, "The replication of DNA in Escherichia coli". Proc. Natl. Acad. Sci. USA, 1958, 44(7), 671682.

${ }^{18}$ H. Jonas, p. 107, op. cit.

${ }^{19}$ H. Jonas, p. 107, op. cit.

${ }^{20}$ X. Su, K.E. Wellen and J.D. Rabinowitz, "Metabolic control of methylation and acetylation", Current Opinion in Chemical Biology, 2016, 30, 52-60.

${ }^{21}$ H. Jonas, p. 107 , op. cit.

${ }^{22}$ On notera que cette remarque rejoint cependant de très près un texte de Jacques Monod qui peut être pris comme représentatif de la manière de penser d'une partie des biologistes moléculaires de cette période (« le système tout entier par conséquent est totalement et intensément conservateur, fermé sur soi-même et absolument incapable de recevoir quelque enseignement que ce soit du monde extérieur. Comme on le voit, ce système par ses fonctionnements d'horlogerie microscopique qui établit entre $\mathrm{ADN}$ et protéines, comme aussi entre organisme et milieu des relations à sens unique, défie toute description "dialectique". Il est foncièrement cartésien et non hégélien : la cellule est bien une machine (J. Monod, Le hasard et la nécessité, Paris, Editions du Seuil, 1970, p.145.). Notons cependant que la perspective jonassienne de la relation de l'organisme à son milieu est orthogonale avec la vue développée par Monod à la fin de ce paragraphe.
} 
une évolution de la biologie, y compris dans ses implications politiques et sociales ${ }^{23}$ ), il existe d'autres mécanismes de modifications chimiques de l'ADN. Par exemple, les cytidine-désaminases modifient les bases cytidines et augmentent le taux de mutations de plusieurs ordres de grandeur dans des situations où l'hypermutation peut être bénéfique (notamment pour conduire à la diversification des immunoglobulines du système immunitaire adaptatif des vertébrés ${ }^{24}$. Chez la paramécie, l'ADN génomique subit un réarrangement massif après chaque division cellulaire, il est amplifié jusqu'à plusieurs centaines de copies par cellule ${ }^{25}$. Les télomères (extrémités des chromosomes) sont l'objet d'un remplacement mais aussi d'une dégradation, même dans les cellules interphasiques (entre deux divisions). On peut aussi mentionner que l'ADN mitochondrial est répliqué dans les cellules en interphase indépendamment de l'ADN nucléaire et qu'il y a ainsi bien, à ce niveau aussi, remplacement métabolique. Il apparaîtrait donc que, de ce point de vue, l'objection - que Jonas se formule à lui-même - puisse être rejetée, l'ADN ne constituant pas un invariant matériel strict, même si on exclut (comme Jonas le fait) la division cellulaire et la mutation spontanée du champ de l'analyse.

Après avoir traité le cas des organismes unicellulaires (et même si ceux-ci ne sont pas explicitement mentionnés dans l'appendice, ils le sont par opposition à ce développement ultérieur, portant sur les organismes multicellulaires), Jonas considère le cas des organismes multicelullaires (explicitement les métazoaires). Jonas fait intervenir en place du remplacement moléculaire, le remplacement cellulaire. Posant que les cellules nerveuses ne sont pas remplacées, celles-ci (et plus spécifiquement leur noyau contenant l'ADN) constitueraient dans le cas des métazoaires un « véritable invariant physique $»^{26}$. La neurobiologie a longtemps posé que la neurogenèse ne se produit que lors du développement, mais il apparaît à présent que le remplacement neuronal se poursuit dans les individus adultes chez de nombreux métazoaires, y compris chez l'homme ${ }^{27}$. Chez ce dernier, il a pu par exemple être établi que 700 nouveaux neurones sont produits chaque jour dans l'hippocampe à l'âge adulte, en datant l'incorporation dans leur $\mathrm{ADN}$ de carbone ${ }^{14} \mathrm{C}$ issu des essais nucléaires atmosphériques de la période de la guerre froide $(1955-1963)^{28}$. Ce type d'étude montre qu'il existe au moins dans certaines zones du cerveau un renouvellement des cellules neuronales dans l'âge adulte ${ }^{29}$.

\footnotetext{
${ }^{23}$ M. Meloni, "Epigenetics for the social sciences: justice, embodiment and inheritance in the postgenomic age", New genetics and Society, 2015, 34(2), 125-151.

${ }^{24} \mathrm{D}$. Fear, "Mechanisms regulating the targeting and activity of activation induced cytidine deaminase", Current Opinion in Immunology, 2013, 25, 619-628.

${ }^{25}$ T. Sonneborn, "Genetics of cellular differenciation : stable nuclear differenciation in eukaryotic unicells" Annual Reviews in Genetics, 1977, 11, 349-367.

${ }^{26}$ H. Jonas, p. 108 , op. cit.

${ }^{27}$ K.A. Sailor, A.F. Schinder and P.M. Lledo, "Adult neurogenesis beyond the niche: its potential for driving brain plasticity", Current Opinion in Neurobiology, 2017, 42, 111-117.

${ }^{28}$ K.L. Spalding, O. Bergmann, K. Alkass, et al., "Dynamics of hippocampal neurogenesis in adult humans", Cell, 2013, 153(6), 1219-1227.

${ }^{29}$ Le postulat que la formation de nouveaux neurones ne peut avoir lieu chez l'adulte remonte notamment aux travaux du neurologue Santiago Ramon y Cajal (L. Colucci-D'Amato, V. Bonavita and U. di Porzio, "The end of the central dogma of neurobiology: stem cells and neurogenesis in adult CNS", Neurological Sciences, 2006, 27, 266-270). Cette position a pu être appelée le dogme central de la neurobiologie par analogie avec le dogme central de la biologie moléculaire énoncé par
} 
Notons également, que chez différents animaux (y compris l'homme), l'ADN génomique des neurones n'est pas strictement invariant dans la mesure où s'y produisent des insertions de rétrotransposons (éléments d'ADN mobiles). Ainsi, on aboutit à un mosaïcisme génétique extrême dans le cerveau ${ }^{30}$. L'activité de ces rétrotransposons est d'ailleurs plus élevée dans le cerveau que dans d'autres organes de manière que, de ce point de vue, le cerveau n'est pas moins mais plus variable que d'autres organes $^{31}$. On peut compléter cette observation dans deux directions. D'une part, il existe des métazoaires dans lesquels il n'y a pas de remplacement cellulaire. Le vers Caenorhabditis elegans présente à l'âge adulte un lignage cellulaire invariant de 959 cellules qui ne sont pas remplacées (sauf dans la lignée germinale). D'autre part, il existe des animaux dont l'ensemble des cellules est remplacé de façon cyclique; ainsi le prochordé marin Botryllus schlosseri se résorbe entièrement et remplace complètement son corps toutes les deux semaines ${ }^{32}$. Le remplacement cellulaire n'est ainsi pas une condition spécifique des cellules neuronales, ni une constante chez tous les organismes. Retenons simplement que les cellules nerveuses (et leur ADN) ne sont pas strictement des invariants.

Jonas dans son texte considère l'existence de foyers physiques invariants qui échapperaient au métabolisme, mais il conclut que ces observations n'altèrent pas de façon significative « le tableau général » établissant que pour le vivant « l'identité vitale est différente de l'identité physique ${ }^{33}$. Si on tient compte des données biologiques cependant, on en vient à nier directement l'existence de tels foyers invariants stricts. Il apparaît ainsi que la thèse générale de Jonas sur la fluence matérielle fondamentale du vivant est parfaitement en accord avec ces données empiriques. Ainsi, l'étrangeté de ce texte réside dans la tentation de Jonas de poser dans l'ADN d'une part, et dans le système nerveux d'autre part, l'existence de noyaux d'identité à contre-courant de sa propre intuition ontologique. Nous oserons peut-être proposer que, devant la radicalité de sa démarche ontologique, Jonas ait transitoirement réinvesti des substances et des formes comme dépositaires de l'individualité, alors que c'est lui-même qui pose fondamentalement l'être comme devenir, comme si un adieu immédiat à tout substantialisme représentait un pas trop audacieux. Le cerveau et l'ADN sont tous deux des objets scientifiques fortement investis à l'époque moderne comme dépositaires d'essences de l'individualité organique. L'argument d'invariance se pose sur l'ADN et le cerveau alors qu'il aurait pu tout aussi

Francis Crick et selon lequel l'information biologique ne circule qu'à sens unique de l'ADN vers les protéines. Le rapprochement de ces deux dogmes est ici significatif, puisque tous les deux sont remis en question (du moins dans leur forme dérivée). Ces deux postulats essentialisent, chacun à leur façon, d'une part l'ADN et d'autre part le système nerveux central comme dépositaires de l'identité individuelle.

${ }^{30}$ J.A. Erwin, M.C. Marchetto and F.H. Gage, "Mobile DNA elements in the generation of diversity and complexity in the brain", Nature Reviews Neuroscience, 2014, 15, 497-506.

${ }^{31}$ La variabilité et la plasticité potentiellement permises par le renouvellement cellulaire et ce type de mosaïcisme génétique pourraient ainsi influencer le cadre de questionnement du problème cerveau/esprit dans les neurosciences actuelles tel que le pose Catherine Malabou (C. Malabou, Que faire de notre cerveau?, Paris, Bayard, 2004).

${ }^{32}$ A. Sanchez Alvarado and S. Yamanaka, "Rethinking differentiation: stem cells, regeneration, and plasticity", Cell, 2014, 157(1), 110-119.

${ }^{33}$ H. Jonas, p. 108, op. cit. 
bien être posé pour d'autres composants et tissus moins investis symboliquement, de ce point de vue, comme le cristallin ou les tendons qui sont également quasi-invariants ${ }^{34}$.

\section{L'individu immédiat}

Quoi qu'il en soit, ce développement permet de mieux saisir la conception de Jonas de l'organisme concret. Nous voudrions en particulier revenir à ce stade sur cette position jonassienne claire qui borne l'individualité entre deux événements reproductifs («cet événement reproductif [...] qui marque respectivement son début et sa fin ») ${ }^{35}$. Il n'est pas aisé d'un point de vue biologique de situer l'existence de l'individualité organique dans l'intervalle qui sépare deux divisions cellulaires, au moins chez certains organismes ${ }^{36}$. On se trouve là dans la difficulté constante qu'il y a, devant la multiplicité des formes et des modalités du vivant, à l'inscrire dans des cadres généraux : «En vain nous poussons le vivant dans tel ou tel de nos cadres. Tous les cadres craquent. Ils sont trop étroits, trop rigides surtout pour ce que nous voudrions y mettre $»^{37}$. La science biologique avoue sans détours son incapacité à établir des catégories aristotéliciennes stables sur les questions de la définition du vivant ${ }^{38}$ ou celle de l'organisme ${ }^{39}$, la solution étant de considérer que, pour son efficace, des catégories de valeur opérationnelles lui suffissent (pour le vivant, l'organisme, l'individu, l'espèce...). En réalité, l'évènement reproductif dont parle Jonas peut aussi être pris comme celui de la reproduction sexuée et, dans ce cas au moins, l'individualité du parent se distingue de celle de sa descendance méiotique (obtenue par reproduction sexuée). On voit en tout cas que, d'une certaine façon, Jonas prend l'organisme individuel sur sa seule face de l'homéostasie; les dimensions de développement, de croissance et de reproduction sont comme évacuées de sa phénoménologie. Il y a bien souci de l'autoperpétuation de soi mais celui-ci ne fait pas intervenir explicitement une liberté nécessiteuse de changer de forme (développement) ou de croître ou de produire de nouveaux individus (reproduction),

\footnotetext{
${ }^{34}$ K.M. Heinemeier, P. Schjerling, J. Heinemeier et al., "Lack of tissue renewal in human adult Achilles tendon is revealed by nuclear bomb ${ }^{14} \mathrm{C}^{\prime \prime}$, The FASEB Journal, 2013, 27, 2074-2079.

${ }^{35}$ H. Jonas, p. 107, op. cit.

${ }^{36} \mathrm{Si}$ la division cellulaire est symétrique, on peut poser que l'individu cellulaire initial ne correspond à aucune des deux cellules produites par celle-ci, et donc que l'existence individuelle est bornée par deux évènements de division. Cependant, si la division cellulaire est asymétrique, comme c'est le cas par exemple chez la levure Saccharomyces cerevisiae, la situation est plus complexe. En effet, la nouvelle cellule (dite improprement cellule fille) bourgeonne à partir de la cellule mère. Il faudrait dès lors considérer que l'existence individuelle de la cellule cesse au moment de l'émergence du bourgeon, au moment où elle devient cellule mère, position difficile à tenir dans la mesure où cette cellule mère est celle qui retient l'essentiel de la mémoire moléculaire de l'expérience métabolique de la cellule initiale. Que dire encore de la situation d'organismes syncytiaux (tels les champignons filamenteux ou les Amoebozoa comme Physarum) dans lesquels les divisions nucléaires ne conduisent pas à des divisions cellulaires proprement dites (pas de cytokinèse). Dans ces cas, l'événement reproductif (pris comme la réplication de l'ADN) ne peut être considéré comme une borne temporelle définissant l'existence individuelle.

${ }^{37}$ H. Bergson, L'évolution créatrice, Paris, Presses Universitaires de France, 1959, p.8.

38 J.C. Letelier, M.L. Càrdenas and A. Cornish-Bowden, "From L'homme machine to metabolic closure: steps towards understanding life", Journal of Theoretical Biology, 2011, 286, 100-113.

39 J.W. Pepper and M.D. Herron, "Does biology need an organism concept?", Biological Reviews 2008, 83, 621-627.
} 
telle que pourrait les inclure une métaphysique schopenhauerienne de la vie subordonnée à la volonté $^{40}$. Simondon considère de manière analogue que «l'individu réel est l'individu mûr, l'individu médian. [...] Jeune et vieux, l'être individué est isolé ; mûr il se structure dans le monde et structure le monde en lui » ${ }^{41}$. Poussée à l'extrême pourtant, cette vue strictement synchronique du vivant pourrait conduire à une difficulté. En effet, si on choisit d'attribuer l'individualité à l'organisme concret hic et nunc, purement somatique, mature, où il n'est que pur métabolisme, pure homéostasie, sans croissance, sans développement, n'arrive-t-on pas à un découpage de l'individualité en une infinité d'individualités temporaires et à la perte de ce que l'on cherchait au premier chef ${ }^{42}$ ? Simondon résout cette difficulté en posant qu'il s'agit de «connaître l'individu à travers l'individuation plutôt que l'individuation à partir de l'individu » ${ }^{43}$ et qu'il convient de faire émerger les questionnements, disons le souci, «des problématiques internes à l'individu». Il écrit «Ce qui devient bonne forme est ce qui, de l'individu, n'est pas encore individué ${ }^{44}$. Dans cette perspective, la liberté nécessiteuse, le souci sont constamment renouvelés, de l'intérieur, même dans des conditions physico-chimiques hypothétiquement stables. Le développement est alors interprété comme des étapes de l'être qui sont des solutions qui résolvent de manière successive des problématiques internes. L'axiomatique, la donation de sens que réalise l'organisme sont ainsi en permanence réévaluées. C'est le souci, le chevauchement de ces temps et ces espaces toujours ouverts à nouveau par le besoin et le désir, qui font la continuité de l'individu et lui évite une réduction à un hic et nunc absolu déterminé par référence externe.

Si cette perspective permet de ressaisir les phénomènes de développement et de croissance en les attribuant à une immanence, reste à s'interroger sur l'inscription historique plus large de l'organisme. Jonas offre une phénoménologie de la vie qui est radicale dans le sens où elle exclut l'aspect diachronique évolutif de la vie de l'organisme concret. Nous avons vu que pour poser l'invariance matérielle de l'ADN, il exclut «l'événement reproductif » de son analyse ontologique et de la même façon la mutation est saisie comme incident (« laissons de côté l'incident anormal de la mutation $\gg)^{45}$. Si la mutation est en effet anormale pour l'organisme, elle est au contraire condition de possibilité pour l'évolution. La maxime de Theodosius Dobzhansky qui illustre la synthèse moderne et

\footnotetext{
${ }^{40}$ M. Henry, De la phénoménologie, Tome I, Phénoménologie de la vie Paris, Presses Universitaires de France, 2003, p.129.

${ }^{41} \mathrm{G}$. Simondon, L'individuation à la lumière des notions de forme et d'information, Grenoble, Million, 2005, p.217.

${ }^{42}$ Le fondateur de la cladistique moderne Willi Hennig en arrive à un tel découpage temporel (théoriquement infinitésimal) de l'individu, notamment pour pouvoir tenir compte des modifications formelles et matérielles radicales qui accompagnent le développement et la croissance (en particulier la métamorphose chez les arthropodes) en introduisant le concept de semaphoront, celui qui porte les caractères (Hennig, Willi, Phylogenic Systematics, Urbana, University of Illinois Press, 1966).

${ }^{43}$ G. Simondon, p.24, op. cit.

${ }^{44}$ G. Simondon, p.205, op. cit.

${ }^{45}$ H. Jonas, p. 107, op. cit.
} 
domine encore la biologie d'aujourd'hui, «nothing in biology makes sense except in the light of evolution» pourrait se détourner avec Le phénomène de la vie en «nothing for the organism makes sense in the light of evolution ». L'individu organique n'a que le souci de lui-même, pas de ce qui le précède, ni de ce qui lui fait suite (c'est-à-dire de son succès évolutif potentiel) ${ }^{46}$. Dans l'essai II du Phénomène de la vie, Jonas comprend et explique fort bien qu'avec le darwinisme, le vivant s'inscrit dans une historicité ${ }^{47}$. Mais il isole strictement ce devenir historique de l'organisme réel. Le darwinisme serait certes un événement philosophique qui inaugure une crise du sujet mais pas un vécu. Le germe ne saurait rien « des vicissitudes de l'histoire du soma $»^{48}$, des joies et des souffrances de ce porteur temporaire des caractères (qui rappelle en ce sens le semaphoront de Hennig). Jonas présente la séparation entre germe et soma comme « une étrange parodie du modèle cartésien des deux substances sans communication entre elles ${ }^{49}$. Dans cette analogie, le germe serait la substance inaltérable et immortelle et le soma le domaine étendu, et une certaine vue génocentrique tend en effet à spiritualiser le matériel génétique et à le mettre à part, comme au-dessus du reste de la matière organique ${ }^{50}$.

Il reste qu'à l'heure actuelle une partie des biologistes de l'évolution appelle à une révision de ce cloisonnement entre soma et germen et de la réduction des organismes à des unités de sélection passives et simples objets de l'évolution ${ }^{51}$. Les tenants de ce cadre théorique désigné extended evolutionary synthesis appellent à la prise en compte des données qui font voir les organismes aussi comme sujets de l'évolution. Les notions d'hérédité élargie (inclusive inheritance, qui réactualise notamment le concept d'hérédité des caractères acquis), de plasticité développementale et de construction de niche conduisent à une réévaluation de la relation des organismes au monde. Dans cette conception, la polarisation de l'organisme dans son milieu et la donation de sens que l'organisme

\footnotetext{
${ }^{46}$ La validité de cette position ontologique qui détache le vivant de son inscription évolutive semble soutenue par l'expérience phénoménale du vivant qu'on peut avoir face à des hybrides stériles (comme des mulets par exemple). Ceci sont perçus comme vivants à part entière indépendamment du fait qu'ils soit des individus non-reproducteurs (J.C. Letelier, M.L. Càrdenas and A. Cornish-Bowden, "From L'homme machine to metabolic closure : steps towards understanding life", Journal of Theoretical Biology, 2011, 286, 100-113).

${ }^{47}$ H. Jonas, p. 51, op. cit.

${ }^{48}$ H. Jonas, p. 64, op. cit.

${ }^{49}$ H. Jonas, p. 63, op. cit.

${ }^{50} \mathrm{Il}$ existe un clivage intradisciplinaire dans la biologie qui est comme un analogue de la séparation que Jonas fait entre le vivant comme organisme concret (soma) et le vivant inséré dans une histoire évolutive (germen). Génétique et biochimie (en particulier l'étude du métabolisme) ont pu être des champs disciplinaires relativement étanches l'un à l'autre. La génétique n'a à proprement parlé pas affaire à l'organisme concret mais s'intéresse à des entités formelles, à des particules d'hérédité que sont les gènes en se désintéressant même pendant longtemps de leur base matérielle. De la même façon, les percées majeures dans la compréhension du métabolisme ne doivent rien à l'approche génétique et travaillent avec le corps concret. Ce clivage descend jusque dans les modèles de l'émergence de la vie qui mettent plus l'accent soit sur le métabolisme soit sur la capacité d'évolution et de reproduction. Ces deux types de modèles sur l'origine de la vie fusionnent à nouveau dans le modèle de RNA world dans lequel les fonctions métabolique et informationnelle s'objectivent dans une même molécule.

${ }^{51}$ Levins Richard and Richard Lewontin, The dialectical Biologist, Cambridge, Harvard University Press, 1985, p. 85-106; K.N. Laland, T. Uller, M.W. Feldman et al., "The extended evolutionary synthesis : its structure, assumptions and predictions", Proceedings of the Royal Society B., 2015, 282(1813) , 2015-1019; P. Godfrey-Smith, "The subject as cause and effect of evolution" Interface focus 2017, 7, 20170022.
} 
effectue débordent sa génération et affectent aussi sa descendance et sa communauté. Dans ce contexte, la liberté nécessiteuse du vivant participe aussi de son inscription dans une historicité. De manière comparable à Jonas sur le fond, mais dans un style différent, Godfrey-Smith base cette subjectivité de l'organisme sur deux éléments, le point de vue et l'intention (point-of-view et agenda)

${ }^{52}$ et invite à la prendre en compte comme force causale dans l'évolution. Les données épigénétiques actuelles montrent aussi que le génome, contre la vision de système «purement catalytique » et « clos », participe à l'évaluation du milieu et à la donation de sens qui est centrale dans l'ontologie du vivant de Jonas.

\section{Vers une téléologie incarnée}

L'organique occupe une position médiane entre deux césures, deux plis : en aval, celle qui articule le vivant au monde physique (la transition du physique au physiologique), en amont, celle qui articule le vivant à l'esprit (la transition du physiologique au psychologique). La porosité que l'on attribue à chacune de ces frontières, le choix de les voir comme discontinuités absolues ou comme transitions progressives détermine pour une grande part les systèmes philosophiques et bien sûr singulièrement ceux ayant trait à la philosophie de la vie. Jonas choisit dans Le phénomène de la vie de marquer le pli entre physique et physiologique et de laisser deviner une continuité entre physiologique et psychologique. Ces césures sont aussi les lieux d'enjeux scientifiques majeurs portant d'une part sur l'origine de la vie, et de l'autre sur l'articulation cerveau/esprit. Ces articulations sont à ce point déterminantes que la biologie moderne a choisi pour l'essentiel d'opter pour une position métaphysique qui assure la continuité du monde physique et du monde vivant, cette position qui pose la Nature comme objective étant prise comme la condition de possibilité d'une science biologique ${ }^{53}$. Certaines reprises récentes de la philosophie de Jonas, en accord avec la tradition phénoménologique, invitent à prendre au contraire la thèse de la subjectivité des organismes (plus que de la Nature) comme position épistémologique fondamentale ${ }^{54}$. La perspective kantienne soutient que la vision téléologique du monde organique est une obligation herméneutique et heuristique mais pas (nécessairement) une réalité. Weber et Varela, en proposant une synthèse de la philosophie de Hans Jonas et de la biologie théorique de l'autopoiesis, proposent la notion de téléologie incarnée (embodied teleology) comme tentative de dépassement de la position kantienne. Peut-être pourrait-on proposer que la vue mécaniciste du vivant et la présentation de la Nature comme objective constituent en fait une épistémologie qui relève, de façon inversée, du als $o b$ kantien. Là où Kant accordait à la vision téléologique de la Nature, une nécessité épistémologique pour aborder la compréhension du

\footnotetext{
${ }^{52}$ P. Godfrey-Smith, op. cit.

${ }_{53}^{53}$ J. Monod, Le hasard et la nécessité, Paris, Editions du Seuil, 1970, p.145.

${ }^{54}$ A. Weber et F.J. Varela, op. cit.
} 
vivant, la représentation d'une Nature objective pourrait être également un comme si : on fait comme si cette Nature était objective pour pouvoir déployer une biologie newtonienne (alors que la Nature se compose en fait d'organismes subjectifs) ${ }^{55}$.

Si la position de Jonas partage avec la Naturphilosophie et l'hégélianisme, l'intention d'éviter une étanchéité entre le monde naturel et le monde de l'esprit, son originalité réside dans sa capacité à remplacer une téléologie idéale par une téléologie immanente et incarnée, spécifique à chaque organisme (individu). Cette subjectivité immanente résulte du souci du vivant qui donne sens à son milieu dans une polarisation entre positivité et négativité. La pertinence de la perspective de Jonas qui embrasse d'un même regard la condition des organismes les plus simples et l'existence humaine trouve son illustration dans la ressemblance entre la figure du Dieu mathématicien de Jonas et celui évoqué dans ces lignes de Merleau-Ponty :

Il y a dans l'existence humaine un principe d'indétermination, et cette indétermination n'est pas pour nous seulement, elle ne vient pas de quelque imperfection de notre connaissance, il ne faut pas croire qu'un Dieu pourrait sonder les cours et les reins et délimiter ce qui nous vient de la nature et ce qui nous vient de la liberté. L'existence est indéterminée en soi, à cause de sa structure fondamentale, en tant qu'elle est l'opération même par laquelle ce qui n'avait pas de sens prend un sens ${ }^{56}$.

De la même façon, le terme de «métabolisme » (si central chez Jonas comme moyen de souligner le changement au cœur de la vie) est aussi utilisé par la philosophe Catherine Malabou quand elle explore la plasticité et les modalités du changement de la conscience. Elle relève que Heidegger propose que le terme Erfahrung - l'expérience vécue - pourrait être traduit par metabolè et souligne que «Penser l'expérience de la conscience comme metabolè implique alors de la comprendre comme arrachement à soi, un lancer de soi qui produit l'identité au lieu de la présupposer ${ }^{57}$, très proche en cela de la position simondienne qui propose de «connaitre l'individu à travers l'individuation ${ }^{58}$ et non l'inverse.

Nous avons tenté ici à partir de la discussion d'un appendice paradoxal du Phénomène de la vie (où Jonas semble se prendre à contre-pied) de souligner que des aspects centraux de son ontologie du vivant ne sont pas contredits par les observations de la biologie positive. Cette analyse nous permet de revenir sur l'importance centrale que revêt pour Jonas l'organisme immédiat par opposition à son

\footnotetext{
${ }^{55}$ R. Levins et R. Lewontin, op. cit.

${ }^{56}$ M. Merleau-Ponty, Euvres, Paris, Gallimard, 2010, p.856.

${ }^{57}$ C. Malabou, La plasticité au soir de l'écriture Paris, Léo Scheer, 2005, p.55.

${ }^{58}$ G. Simondon, p.24, op. cit.
} 
inscription évolutive. Peut-être est-il possible de souligner que l'ontologie du vivant posée par Jonas permet par sa simplicité, son caractère minimaliste d'inclure dans une même perspective tout le vivant, des micro-organismes procaryotes jusqu'à l'homme, en se centrant sur l'individu et sa subjectivité. Il existe historiquement une interface entre phénoménologie et neurosciences (même si celle-ci est parfois un lieu polémique $)^{59}$. La position jonassienne laisse, nous semble-t-il, aussi une possibilité d'articuler de manière plus générale les démarches phénoménologique et biologique (une possibilité que n'offrent peut-être pas des perspectives vitalistes comme celle de Bergson par exemple). Nous soutiendrons donc que son ontologie est actuelle et pourrait être utile aujourd'hui pour penser notamment la question des relations humains/non-humains ${ }^{60}$. Elle raisonne clairement avec la prise en compte présente, élargie, de la subjectivité des organismes, que celle-ci soit considérée de manière générale dans les théories évolutives ${ }^{61}$ ou plus spécifiquement dans les approches éthologiques de la subjectivité animale ${ }^{62}$.

\footnotetext{
${ }^{59}$ W. Feuerhahn, "Un tournant neurocognitiviste en phénoménologie ? Sur l'acclimatation des neurosciences dans le paysage philosophique français ", Revue d'Histoire des Sciences Humaines 2011, 25 59-79.

${ }^{60}$ D. Haraway, "Anthropocene, Capitalocene, Plantationocene, Chthulucene: Making kin." Environmental Humanities, 2015, 6, 159-165.

${ }^{61}$ P. Godfrey-Smith, op. cit.

${ }^{62}$ R. Marchesini, "Philosophical ethology and animal subjectivity", Angelaki 2016, 21(1), 237-252.
} 